\title{
Exact bounds on epsilon processes
}

\author{
Toshiyasu Arai \\ Graduate School of Science, Chiba University \\ 1-33, Yayoi-cho, Inage-ku, Chiba, 263-8522, JAPAN
}

\begin{abstract}
In this paper we show that the lengths of the approximating processes in epsilon substitution method are calculable by ordinal recursions in an optimal way.
\end{abstract}

Epsilon substitution method is a method proposed by D. Hilbert to prove the consistency of (formal) theories. The idea behind the method is that one could replace consistently transfinite/non-computable objects as a figure of speech by finitary/computable ones as far as transfinite ones are finitely presented as axioms of a theory. In other words, the replacement (epsilon substitution) depends on contexts, i.e., formal proofs in which axioms for the transfinite objects occur. If this attempt would be successfully accomplished, then the (1-)consistency of the theory follows.

For example, for first order arithmetic PA, replace each existential formula $\exists x F[x]$ by $F[\epsilon x . F[x]]$, where the epsilon term $\epsilon x . F[x]$ intends to denote the least number satisfying $F[x]$ if such a number exists. Otherwise it denotes an arbitrary object, e.g., zero. Then PA is interpretable in an extended 'propositional calculus' having the epsilon axioms:

$$
\text { ( } \epsilon) F[t] \rightarrow \epsilon x . F[x] \ngtr t \wedge F[\epsilon x . F[x]]
$$

The problem is to find a solving substitution which assigns numerical values to epsilon terms and under which all the epsilon axioms occurring in a given proof are true.

Hilbert's Ansatz is, starting with the null substitution $S^{0}$ which assigns zero to whatever, to approximate a solution by correcting false values step by step, and thereby generate the process $S^{0}, S^{1}, \ldots$ (H-process). The problem is to show that the process terminates.

In [2, 3], 4, [5] and 7], we formulated H-processes for theories of jump hierarchies, for $I D_{1}\left(\Pi_{1}^{0} \vee \Sigma_{1}^{0}\right)$, for $\left[\Pi_{1}^{0}, \Pi_{1}^{0}\right]$-FIX, for $\Pi_{1}^{0}$-FIX and for $\Pi_{2}^{0}$-FIX, resp., and proved that the processes terminate by transfinite induction up to the relevant proof-theretic ordinals.

In this paper we address a problem related to these termination proofs, and show that the lengths of the processes are calculable by ordinal recursions in an optimal way. 
Let $\mathrm{T}$ denote one of the following theories; first order arithmetic, the theories of the absolute jump hierarchy, theories $\Phi$-FIX for non-monotonic inductive definitions for the formula classes $\Phi=\Pi_{1}^{0},\left[\Pi_{1}^{0}, \Pi_{1}^{0}\right], \Pi_{2}^{0}$. Let $|T|$ denote the proof-theoretic ordinal of $\mathrm{T}$.

Given a finite sequence $C r$ of critical formulas, let $\left\{S^{n}\right\}$ denote the H-process for $\mathrm{Cr}$.

Theorem 1 The length $H=\min \left\{n: S^{n}\right.$ is a solution $\}$ of the H-process up to reaching a solution for $\mathrm{Cr}$, is calculable by $|T|$-recursion.

Therefore so is the solution $S^{H}$.

\section{First order arithmetic: Ackermann's proof}

In this section we give the ordinal-theoretic heart of the epsilon substitution method.

\subsection{The H-process}

The language of first order arithmetic PA includes some symbols for computable functions, say + for addition, - for multiplication and - for cut-off subtraction, and the relation symbol $<$. In its $\epsilon$-counterpart $\mathrm{PA} \epsilon$, formulas and terms are defined simultaneously by stipulating that

$$
\text { if } F \text { is a formula, then } \epsilon x . F \text { is a term. }
$$

By expression we mean a term or a formula.

An $\epsilon$-substitution $S$ is a finite function assigning values $|\epsilon x . F|_{S} \in \omega$ of canon$i c a l(=$ closed and minimal epsilon) terms $\epsilon x . F . \operatorname{dom}(S)$ denotes its domain.

$\epsilon$-substitutions $S$ reduces an expression $e$ to its unique irreducible form $|e|_{S}$ by using default value 0 for expressions not in $\operatorname{dom}(S)$.

Let $C r=\left\{C r_{0}, \ldots, C r_{N}\right\}$ be a fixed finite sequence of closed epsilon axioms. $S$ is solving if $S$ validates any critical formula in $C r$. Otherwise $S$ is nonsolving.

The existence of a solving substitution for any finite sequence of critical formuls yields the 1-consistency of PA.

The $\operatorname{rank} r k(e)<\omega$ of an expression $e$ measures nesting of bound variables in $e$.

Definition $2 r k(S):=\max (\{r k(e): e \in \operatorname{dom}(S)\} \cup\{0\})$.

For a substitution $S$ and a natural number $r, S_{<r}:=\{(e, v) \in \operatorname{Srk}(e)<r\}$.

For a fixed sequence $C r$, the $H$-process $S^{0}(=\emptyset), S^{1}, \ldots$ of substitutions for $\mathrm{Cr}$ is defined using the ranks of $\epsilon$-terms. The sequence $\left\{S^{n}\right\}$ is primitive (or even elementary) recursive. We assume that if $S^{n}$ is a solution for $\mathrm{Cr}$, then $S^{m}=S^{n}$ for any $m \geq n$.

By an algorithm, we associate an epsilon axiom $\operatorname{Cr}(S)$ to a nonsolving substitution $S$ :

$$
C r(S): F[t] \rightarrow \epsilon x . F[x] \ngtr t \wedge F[\epsilon x . F[x]],
$$


which is false under $S$. Then $e^{S}: \equiv \epsilon x .|F|_{S}$ and $v^{S}:=|t|_{S}$.

If $S^{n}$ is nonsolving, then the next substitution is defined as follows.

$$
S^{n+1}:=S_{<r k\left(e^{S^{n}}\right)}^{n} \cup\left\{(f, u) \in S^{n}: r k(f)=r k\left(e^{S^{n}}\right) \& f \not \equiv e^{S^{n}}\right\} \cup\left\{\left(e^{S^{n}}, v^{S^{n}}\right)\right\} .
$$

\subsection{Termination proof}

In this subsection we recall a proof of the termination of the H-process. The proof is based on the transfinite induction up to $\varepsilon_{0}$.

Define the Ackermann ordering:

$$
x<_{A} y: \Leftrightarrow[x \neq 0 \& y=0] \vee[x, y \neq 0 \& x<y]
$$

Thus 0 is the largest element in $<_{A}$. $\|x\|_{A}$ denotes the order type of $x$ in the ordering $<_{A}$.

A relation $T \sqsubseteq_{A} S$ on $\epsilon$-substitutions is defined.

\section{Definition 3}

$$
\begin{aligned}
T \sqsubseteq_{A} S & : \Leftrightarrow \quad \forall(e, u) \in S \exists(e, v) \in T\left[v \leq_{A} u\right] \\
& \Leftrightarrow \quad|e|_{T} \leq_{A}|e|_{S} \text { for any canonical } e
\end{aligned}
$$

We associate an ordinal $\operatorname{ind}(S)<\omega^{\omega}($ index of $S$ ) relative to a fixed sequence $\mathrm{Cr}$ of $\epsilon$-axioms.

$C l_{\epsilon}(\mathrm{Cr})$ denotes the set of closed $\epsilon$-terms occurring in the set $\mathrm{Cr}$. Let $N(\mathrm{Cr}):=\# C l_{\epsilon}(\mathrm{Cr})\left(=\right.$ the cardinality of the set $\left.C l_{\epsilon}(\mathrm{Cr})\right) . N(\mathrm{Cr})$ is less than or equal to the total number of occurrences of the symbol $\epsilon$ in the set $C r$.

Definition 4 1. For an $e \in C l_{\epsilon}(C r)$ put

$$
\varphi(e ; S):=\|v\|_{A} \text { for } v=|e|_{S} .
$$

2. We arrange the set $C l_{\epsilon}(C r)$ of cardinality $N(C r)$ as follows: $C l_{\epsilon}(C r)=$ $\left\{e_{i}: i<N(C r)\right\}$ where

$$
e_{j} \text { is a closed subexpression of } e_{i} \Rightarrow j>i
$$

3.

$$
\operatorname{ind}(S)=\sum\left\{(\omega+1)^{i} \cdot \varphi\left(e_{i} ; S\right): i<N(C r)\right\}
$$

Let IND $:=\operatorname{IND}(C r):=(\omega+1)^{N(C r)}$.

Let $r_{n}=r k\left(S^{n}\right), e_{n}=e^{S^{n}}, v_{n}=v^{S^{n}}$ and $a_{n}=\operatorname{ind}\left(S^{n}\right)$ up to a solution. Otherwise let $r_{n}=e_{n}=v_{n}=a_{n}=0$.

The epsilon axiom $\operatorname{Cr}(S)$ associated to nonsolving substitutions $S$ depends only on their indices $\operatorname{ind}(S)$.

Lemma 5 (Cf. [5])

Let $S^{n}$ and $S^{m}$ be nonsolving substitutions such that $S^{m} \sqsubseteq_{A} S^{n}$. Then 
1. $a_{n} \geq a_{m}$.

2. $S^{m+1} \sqsubseteq_{A} S^{n+1} \& e_{n}=e_{m} \& v_{n}=v_{m}$ and $r_{n+1}=r_{m+1}$ if $a_{n}=a_{m}$.

Each $S^{n}$ is shown to be correct, cf. [5]. This yields the following fact for nonsolving $S^{n}$.

$$
\left(e_{n}, v\right) \in S^{n} \Rightarrow 0 \neq v_{n}<v
$$

Fix a positive integer RANK $=\operatorname{RANK}(C r):=\max \{r k(C r)+1,2\}$, where $r k(C r):=\max \left\{r k\left(C r_{I}\right): I=0, \ldots, N\right\}$. Then for any $S$ appearing in the $\mathrm{H}$-process, we have $r k(S)<\mathrm{RANK}$.

Let

$$
\vec{S}^{m, k}=\left\{S^{n}\right\}_{m \leq n<k} .
$$

Definition 6 Let $\vec{S}^{m, k}$ be a consecutive series in the H-process $S^{0}, \ldots$ Then

$$
r k\left(\vec{S}^{m, k}\right):=\min \left(\left\{r_{i}: m<i<k\right\} \cup\{\mathrm{RANK}\}\right)>0 .
$$

Definition 7 A consecutive series $\vec{S}^{m, k}$ in the H-process $S^{0}, \ldots$ is a section iff $r_{m}<r k(\vec{S})$.

Definition 8 Let $\vec{S}^{i}=\vec{S}^{m^{i}, k^{i}}(i=0,1)$ be two consecutive series in the Hprocess $S^{0}, \ldots$ such that $r_{m^{i}} \leq r k\left(\vec{S}^{i}\right)$ for $i=0,1$.

If $S^{m^{1}} \sqsubseteq_{A} S^{m_{0}}$ and one of the following conditions is fulfilled, then we write $\vec{S}^{1} \prec \vec{S}^{0}$ :

1. There exists a $p<\min \left\{\ell^{0}, \ell^{1}\right\}\left(\ell^{i}:=k^{i}-m^{i}\right)$ such that $a_{m^{0}+p}>a_{m^{1}+p}$ and $\forall i<p\left(a_{m^{0}+i}=a_{m^{1}+i}\right)$.

2. $\ell^{1}<\ell^{0}$ and $\forall i<\ell^{0}\left(a_{m^{0}+i}=a_{m^{1}+i}\right)$.

The following Lemma 9 is seen readily from Lemma 5 and (3), cf. [5].

Lemma 9 Let $\vec{S}^{i}=\vec{S}^{m^{i}, k^{i}}(i=0,1)$ be two sections in the H-process $S^{0}, \ldots$ such that $k^{0}=m^{1}$ and $r_{m^{0}} \leq r_{m^{1}}<r k\left(\vec{S}^{0}\right)$. Then

1. $S^{m^{1}} \sqsubseteq{ }_{A} S^{m^{0}}$.

2. $\vec{S}^{1} \prec \vec{S}^{0}$.

Lemma 922 means that each section $\vec{S}=\left\{S_{i}: i \leq k\right\}$ codes an ordinal $o(\vec{S})<\varepsilon_{0}$ in Cantor normal form with base 2: Let $r k\left(S_{0}\right) \leq r k(\vec{S})=: r$. Divide $\vec{S}$ into substrings which are sections as follows. Put $\left\{k_{0}<\cdots<k_{l}\right\}=\{i: i \leq$ $\left.k \& r k\left(S_{i}\right)=r\right\} \cup\{0\}$, and $\vec{S}=\vec{S}_{0} * \cdots * \vec{S}_{l}$ with $\vec{S}_{j}=\left(S_{k_{j}}, \ldots, S_{k_{j+1}-1}\right)$ for $0 \leq j \leq l$ and $k_{l+1}=k+1$.

The series $\vec{S}_{0}, \ldots, \vec{S}_{l}$ of substrings of $\vec{S}$ is called the decomposition 11 of $\vec{S}$.

We have $\forall j<l\left[\vec{S}_{j+1} \prec \vec{S}_{j}\right]$.

\footnotetext{
${ }^{1}$ Note that the definition of the decomposition here differs from one in Definition 12
} 
For ordinals $a$ and $\alpha \geq 2$ and $k<\omega$, let $\alpha_{0}(a):=a$ and $\alpha_{1+k}(a):=\alpha^{\alpha_{k}(a)}$. Also set $\omega_{k}:=\omega_{k}(1)$.

For each series $\vec{S}=\vec{S}^{m, k}$ with $r_{m} \leq r k(\vec{S})$ and a natural number $\xi$ such that $0<\xi \leq r=r k(\vec{S})$, associate an ordinal $o(\vec{S} ; \xi)<\omega_{\mathrm{RANK}+2-\xi}$ so that the following Lemma 10 holds, cf. 2 for a full definition and a proof.

Lemma 10 Let $\vec{S}^{i}=\vec{S}^{m^{i}, k^{i}}(i=0,1)$ be two series in the H-process $S^{0}, \ldots$ such that $\vec{S}^{1} \prec \vec{S}^{0}$ and $r_{m^{i}}<\operatorname{rk}\left(\vec{S}^{i}\right)$ for $i=0,1$.

Then $o\left(\vec{S}^{0} ; \xi\right)>o\left(\vec{S}^{1} ; \xi\right)$ for any natural number $\xi \leq \min \left\{r k\left(\vec{S}^{0}\right), r k\left(\vec{S}^{1}\right)\right\}$.

Theorem 11 (Transfinite induction up to $\varepsilon_{0}$ )

The H-process $S^{0}, \ldots$ terminates.

Proof. Suppose the H-process $S^{0}, \ldots$ is infinite and put $r_{n}=r k\left(S^{n}\right)$.

Inductively we define a sequence $\left\{n_{i}: i \in \omega\right\}$ of natural numbers as follows. First set $n_{0}=0$. Suppose $n_{i}$ has been defined. Then put $\beta_{i}=\min \left\{r_{n}: n>n_{i}\right\}$ and $n_{i+1}=\min \left\{n>n_{i}: r_{n}=\beta_{i}\right\}$.

Then Lemma 10 yields an infinite decreasing sequence of ordinals, viz.

$\forall i\left[o\left(\vec{S}_{i+1} ; \xi\right)<o\left(\vec{S}_{i} ; \xi\right)<\omega_{\mathrm{RANK}}<\varepsilon_{0}\right]$ for $\vec{S}_{i}=\vec{S}^{n_{i}, n_{i+1}}$ and $\xi=\beta_{0}+1 \geq 2$.

Therefore the H-process $S^{0}, \ldots$ for any given sequence $\mathrm{Cr}$ of critical formulas terminates. It provides a closed and solving substitution, which in turn yields the 1-consistency $\mathrm{RFN}_{\Sigma_{1}^{0}}(\mathrm{PA})$ of PA stating that any PA-provable $\Sigma_{1}^{0}$-sentence is true.

However the above proof is not entirely satisfactory. Specifically the 1consistency of PA is known to be equivalent, over a weak arithmetic, to the principle $\mathrm{PRWO}_{\varepsilon_{0}}$, which says that there is no infinite primitive recursive descending chain of ordinals $<\varepsilon_{0}$, or to be equivalent to the totality of $\varepsilon_{0}$-recursive functions. The sequence $\left\{n_{i}\right\}_{i}$ and hence the sequence $\left\{o\left(\vec{S}_{i} ; \xi\right)\right\}_{i}$ of ordinals in the above proof are not seen to be recursive. Therefore we need to show that the sequence $\left\{n_{i}\right\}_{i}$ is $\varepsilon_{0}$-recursive in showing the 1-consistency of PA.

\section{Exact bounds: finite ranks}

In this section we show that the length of the H-process up to reaching a solution is bounded by an ordinal recursive function. From the bound one can easily read off the bound for the provably recursive functions in PA.

\subsection{Ordinal recursive functions}

Let us recall the definition and facts on ordinal recursive functions in $\mathrm{W}$. W. Tait 8 .

Let $<_{\Lambda}$ denote a primitive recursive well ordering of type $\Lambda>0$. Assume that 0 is the least element in $<_{\Lambda}$.

For each $\alpha \leq \Lambda,<_{\alpha}$ denotes the initial segment of $<_{\Lambda}$ of type $\alpha$. A numbertheoretic function is said to be $\alpha$-recursive iff it is generated from the schemata 
for primitive recursive functions plus the following schema for introducing a function $f$ in terms of functions $g, h$ and $d$ :

$$
f(\vec{y}, x)= \begin{cases}g(\vec{y}, x) & \text { if } d(\vec{y}, x) \nless_{\alpha} x \\ h(\vec{y}, x, f(\vec{y}, d(\vec{y}, x))) & \text { if } d(\vec{y}, x)<_{\alpha} x\end{cases}
$$

A function is $<\alpha$-recursive iff it is $\beta$-recursive for some $\beta<\alpha$.

W. W. Tait 8 , p.163 shows that for each $\alpha$ the class of $\alpha$-recursive functions is closed under the external recursion to introduce a function $f$ in terms of functions $g, h, d$ and $e$ :

$$
f(\vec{y}, x)= \begin{cases}g(\vec{y}, x) & \text { if } e(\vec{y}, d(\vec{y}, x)) \nless_{\alpha} e(\vec{y}, x) \\ h(\vec{y}, x, f(\vec{y}, d(\vec{y}, x))) & \text { if } e(\vec{y}, d(\vec{y}, x))<_{\alpha} e(\vec{y}, x)\end{cases}
$$

\section{$2.2 p$-series}

In this subsection we define a series $\vec{S}^{m, k}$ to be a $p$-series. $p$-series is introduced for counting the number of ranks $r_{n}$ in the $\mathrm{H}$-process.koko

Given the finite sequence $C r=\left\{C r_{I}: I \leq N\right\}$ of critical formulas in PA $\epsilon$, let $\left\{S^{n}\right\}$ denote the H-process for $C r$. Recall that the sequence is infinite in the sense that if $S^{n}$ is a solution for $C r$, then $S^{m}=S^{n}$ for any $m \geq n$.

Recall that $\omega^{\omega}>\operatorname{IND}=\operatorname{IND}(C r):=(\omega+1)^{N(C r)}>a_{n}$ and $\omega>$ RANK $=$ $\operatorname{RANK}(C r)>r_{n}$ for any $n$.

For $m<k$ let

$$
\begin{aligned}
\operatorname{nd}\left(\vec{S}^{m, k}\right) & :=\left\{n \in[m, k): r_{n} \leq r k\left(\vec{S}^{n, k}\right)\right\} \\
& \left(=\left\{n \in[m, k): r_{n}=\min \left(r_{i}: i \in[n, k)\right)\right\}\right) .
\end{aligned}
$$

Definition 12 Let $\vec{S}=\vec{S}^{m, k}$ (with $m<k$ ) such that $r_{m} \leq r k(\vec{S})$ (i.e., $m \in$ $\operatorname{nd}(\vec{S}))$, and let $\left\{k_{0}, \ldots, k_{l}\right\}_{<}=\operatorname{nd}(\vec{S})$. Then $\left(\vec{S}_{0}, \ldots, \vec{S}_{l}\right)$ with $\vec{S}_{j}:=\vec{S}^{k_{j}, k_{j+1}}$ and $k_{l+1}:=k$ is called the decomposition of $\vec{S}$ into substrings. Each substring $\vec{S}_{j}(0 \leq j \leq l)$ is called a component in the decomposition of $\vec{S}$

Note that $k_{0}=m, k_{l}=k-1$, and $r k\left(S^{k_{j}}\right) \leq r k\left(S^{k_{j+1}}\right)<r k\left(\vec{S}_{j}\right)$ for $j<l$. Also note that each component $\vec{S}_{j}$ is a section.

Lemma 13 Let $\vec{S}^{i}=\vec{S}^{m^{i}, k^{i}}(i=0,1)$ with $k^{0}=m^{1}$ such that $r_{m^{i}} \leq r k\left(\vec{S}^{i}\right)(i=$ $0,1)$ and $r_{m^{0}} \leq r_{m^{1}}$. Then for $\vec{S}:=\vec{S}^{0} * \vec{S}^{1}$ we have

$$
\operatorname{nd}(\vec{S})=\left\{n \in \operatorname{nd}\left(\vec{S}^{0}\right): n \leq I\right\} \cup \operatorname{nd}\left(S^{1}\right),
$$

where $I:=\max \left\{n \in\left[m^{0}, k^{0}\right): r_{n} \leq r_{m^{1}}\right\}$.

Proof. We see $I=k_{J}^{0}$ for a $J \leq l^{0}$ from the facts that both $\left\{S^{n}: I \leq n<m^{1}\right\}$ and each $\vec{S}_{j}^{0}\left(j \leq l^{0}\right)$ are sections and $k_{l^{0}}^{0}=m^{1}-1$. Therefore $m^{1}=k_{0}^{1}=k_{J+1}$ and the lemma is shown. 
Definition 14 Let $\vec{S}=\vec{S}^{m, k}$ with $m<k$ such that $r_{m} \leq r k(\vec{S})$. Define inductively the series $\vec{S}$ to be a p-series and a p-section as follows:

1. $\vec{S}$ is a 0 -series iff $k=m+1$, i.e., a singleton.

2. A $p$-series is a $p$-section iff it is a section.

3. Let $\vec{S}=\vec{S}_{0} * \cdots * \vec{S}_{l}$ be the decomposition of $\vec{S}$ into substrings. Then $\vec{S}$ is a $(p+1)$-series iff each substring $\vec{S}_{j}$ is a $p$-section, or equivalently a $p$-series.

\section{Lemma 15 1. Each $p$-series is a $(p+1)$-series.}

2. Let $\vec{S}^{i}=\vec{S}^{m_{i}, k_{i}}(i=0,1)$ be two p-series overlapped, i.e., $\left[m_{0}, k_{0}\right) \cap$ $\left[m_{1}, k_{1}\right) \neq \emptyset$. Then the union $\vec{S}=\vec{S}^{\min \left\{m_{0}, m_{1}\right\}, \max \left\{k_{0}, k_{1}\right\}}$ is a p-series.

3. Let us call a $p$-series proper if $p=0$, or $p>0$ and it is not $a(p-1)$-series.

(a) If $\vec{S}$ is a proper $p$-section, then

$$
\#\{r k(S): S \in \vec{S}\} \geq p+1 .
$$

(b) If $\vec{S}$ is a proper $p$-series, then

$$
\#\{r k(S): S \in \vec{S}\} \geq p .
$$

(c) If a proper p-series $\vec{S}$ begins with $S^{0}=\emptyset$, then

$$
\#\{r k(S): S \in \vec{S}\} \geq p+1 .
$$

Therefore there is no proper RANK-series beginning with $S^{0}$.

Proof. By induction on $p$.

[1511. A 0 -series $\left\{S^{n}\right\}$ is a 1 -series.

1512. Assume $p>0$ and one is not a substring of the other, i.e., $\left[m_{i}, k_{i}\right) \nsubseteq$ $\left[m_{1-i}, k_{1-i}\right)$. Then without loss of generality we may assume $m_{0}<m_{1}<$ $k_{0}<k_{1}$. Decompose the $p$-series $\vec{S}^{i}$ to the sequence of $(p-1)$-series $\vec{S}_{j}^{i}=$ $\left(S^{k_{j}^{i}}, \ldots, S^{k_{j+1}^{i}-1}\right)\left(j \leq l_{i}\right)$. It suffices to show: $m_{1} \leq k_{j}^{i} \leq k_{0} \Rightarrow \exists j^{\prime}\left(k_{j}^{i}=k_{j^{\prime}}^{1-i}\right)$.

This is seen from the condition that each decomposition $\left\{\vec{S}_{j}^{i}: j \leq l_{i}\right\}$ is a sequence of sections with nondecreasing ranks of the first terms.

15|3. Let $\vec{S}=\vec{S}_{0} * \cdots * \vec{S}_{l}$ be a proper $(p+1)$-series with $\operatorname{nd}(\vec{S})=\left\{k_{0}, \ldots, k_{l}\right\}_{<}$. Then $l>0$ and one of $p$-sections $\vec{S}_{j}$ is proper. Lemma 1513a yields $\#\{r k(S)$ : $\left.S \in \vec{S}_{j}\right\} \geq p+1$, and hence Lemma 153 b follows.

If $\vec{S}_{0}$ is proper, then $r_{k_{0}}<r_{k_{1}}<r k\left(\vec{S}_{0}\right)$ since $\vec{S}_{0}$ is a section. Hence $\#\left(\left\{r k(S): S \in \vec{S}_{0}\right\} \cup\left\{r_{k_{1}}\right\}\right) \geq p+2$. Next assume $j>0$. Then $r_{k_{0}}<r_{k_{j}}<$ $r k\left(\vec{S}_{j}\right)$, and $\#\left(\left\{r k(S): S \in \vec{S}_{j}\right\} \cup\left\{r_{k_{0}}\right\}\right) \geq p+2$. This shows Lemma 1513a,

Lemma $15 / 3 \mathrm{C}$ is seen from the fact $r_{n}>0$ for $n>0$. Namely any proper $p$-series $\vec{S}$ beginning with $S^{0}=\emptyset$ is a section. 
Lemma 16 Let $\vec{S}^{i}=\vec{S}^{m^{i}, k^{i}}(i=0,1)$ be two consecutive series, $k^{0}=m^{1}$ such that $r_{m^{i}} \leq r k\left(\vec{S}^{i}\right)(i=0,1)$ and $r_{m^{0}} \leq r_{m^{1}}$.

The concatenated series $\vec{S}=\vec{S}^{m_{0}, k_{1}}$ is a $(p+1)$-series if $\vec{S}^{0}$ is a p-series and $\vec{S}^{1}$ is a $(p+1)$-series.

Proof. This is seen from Lemmas 13 and 15ा1.

Let $<_{\varepsilon_{0}}$ denote a standard well ordering of type $\varepsilon_{0}$ with the least element 0 .

Lemma 17 Let $\vec{S}^{i}=\vec{S}^{m^{i}, k^{i}}(i=0,1)$ be two $p$-series such that $k^{0}=m^{1}, S^{k^{1}-1}$ is nonsolving and $r_{m^{0}} \leq r_{m^{1}}$. For $o\left(\vec{S}^{i}\right):=o\left(\vec{S}^{i} ; 0\right)$ we have $o\left(\vec{S}^{1}\right)<_{\varepsilon_{0}} o\left(\vec{S}^{0}\right)$.

Proof. By Lemma 10 it suffices to show $\vec{S}^{1} \prec \vec{S}^{0}$. As in Lemma 9 this is seen as follows. Let $\ell^{i}:=k^{i}-m^{i}$.

Since the relation $\sqsubseteq_{A}$ is transitive, we have $S^{m^{1}} \sqsubseteq_{A} S^{m^{0}}$ by Lemma 911 Using Lemma 5, it suffices to show that the following case never happen: $\ell^{0} \leq \ell^{1}$ and $\forall i<\ell^{0}\left[a_{m^{0}+i}=a_{m^{1}+i}\right]$.

If this happens, then we would have $a_{m^{1}-1}=a_{m^{0}+\ell^{0}-1}=a_{m^{1}+\ell^{0}-1}$, and hence $S^{m^{1}} \ni\left(e_{m^{1}-1}, v_{m^{1}-1}\right)=\left(e_{m^{1}+\ell^{0}-1}, v_{m^{1}+\ell^{0}-1}\right)$ by Lemma [5]2. On the other hand we have $S^{m^{1}+\ell^{0}-1} \sqsubseteq_{A} S^{m^{1}}$ by Lemma 91.

For a $v \leq_{A} v_{m^{1}+\ell^{0}-1}$ we would have $\left(e_{m^{1}+\ell^{0}-1}, v\right) \in S^{m^{1}+\ell^{0}-1}$. By (3) we have $0 \neq v_{m^{1}+\ell^{0}-1}<v$. A contradiction.

$k=M(p, n)$ defined below will denote the number such that $\vec{S}^{n, k}$ is the longest $p$-series starting with nonsolving $S^{n}$.

Definition $18 M(0, n):=n+1$.

Case $0 S^{n}$ is solving: $M(p+1, n):=n$.

Case $1 S^{n}$ is nonsolving. Let

$$
e_{p}(n):=o\left(\vec{S}^{n, M(p, n)}\right)
$$

Then define

$$
M(p+1, n):= \begin{cases}M(p, n) & \text { if } e_{p}(M(p, n)) \nless_{\varepsilon_{0}} e_{p}(n) \\ M(p, n) & \text { if } r_{M(p, n)}<r_{n} \& e_{p}(M(p, n))<_{\varepsilon_{0}} e_{p}(n) \\ M(p+1, M(p, n)) & \text { if } r_{M(p, n)} \geq r_{n} \& e_{p}(M(p, n)) \varepsilon_{\varepsilon_{0}} e_{p}(n)\end{cases}
$$

Actually the function $M(p, n)$ depends also on the given sequence $\mathrm{Cr}$ of epsilon axioms. We write $M(p, n ; C r)$ for $M(p, n)$ when the parameter $C r$ should be mentioned.

A consecutive series $\vec{S}^{n, k}$ is a normal $p$-series iff it is a $p$-series and $S^{k-1}$ is nonsolving if $k>n$.

Lemma 19 1. If $S^{n}$ is nonsolving, then $\vec{S}^{n, M(p, n)}$ is a normal p-series.

2. If $\vec{S}^{n, k}$ is a normal p-series, then $k \leq M(p, n)$. 
3. $S^{H}$ is a solution for $C r$, where $H=H(C r):=M($ RANK $-1,0 ; C r)$.

\section{Proof.}

[191. Main induction on $p$. The case when $p=0$ is trivial.

The case $p+1$ is proved by side induction on $e_{p}(n)$. Assume that $S^{n}$ is nonsolving.

1. $M(p+1, n)=M(p, n)$ : Then by $\mathrm{MIH}(=$ Main Induction Hypothesis) $\vec{S}^{n, M(p, s)}$ is a normal $p$-series. $\vec{S}^{n, M(p, s)}$ is also a normal $(p+1)$-series by Lemma 15ा1.

2. $M(p+1, n) \neq M(p, n)$ : Then with $k=M(p, n)$ we have $r_{k} \geq r_{n}$ and $M(p+$ $1, n)=M(p+1, k)$. By MIH $\vec{S}^{n, k}$ is a normal $p$-series. Since $M(p+1, k) \neq$ $k, S^{k}$ is nonsolving, and hence by $\mathrm{MIH}, \vec{S}^{k, M(p, k)}$ is a normal $p$-series. Lemma 17 yields $e_{p}(n)=o\left(\vec{S}^{n, k}\right)>o\left(\vec{S}^{k, M(p, k)}\right)=e_{p}(k)$. Therefore $\vec{S}^{k, M(p+1, k)}$ is a normal ( $\left.p+1\right)$-series by $\mathrm{SIH}$ (=Side Induction Hypothesis). Together with $r_{n} \leq r_{k}$ it follows from Lemma 16 that $\vec{S}^{n, M(p+1, n)}$ is a normal $(p+1)$-series.

192. Main induction on $p$. The case when $p=0$ is trivial.

The case $p+1$. First we show the following:

$$
n \leq n^{\prime}<M(p, n) \Rightarrow M\left(p, n^{\prime}\right) \leq M(p, n)
$$

Assume $n \leq n^{\prime}<M(p, n)=: k$ and $n^{\prime}<M\left(p, n^{\prime}\right):=k^{\prime}$. Then by Lemma 1911 $\vec{S}^{n, k}$ and $\vec{S}^{n^{\prime}}, k^{\prime}$ are two normal $p$-series overlapped. By Lemma 15$] 2$ the union $\vec{S}^{n, \max \left\{k, k^{\prime}\right\}}$ is a normal $p$-series too. By MIH it follows that $k^{\prime} \leq M(p, n)$. This shows (4).

Now by side induction on $k-n$ we prove:

$$
\text { If } \vec{S}^{n, k} \text { is a normal }(p+1) \text {-series, then } k \leq M(p+1, n) \text {. }
$$

Assume that $\vec{S}^{n, k}$ is a normal $(p+1)$-series, and $\operatorname{nd}\left(\vec{S}^{n, k}\right)=\left\{k_{0}, \ldots, k_{l}\right\}_{<}$, $l>0$. Then by MIH we have $k_{1} \leq M(p, n)$. Let $j \leq l$ denote maximal such that $k_{j} \leq M(p, n)$.

1. $k_{j}=M(p, n)$ : Since $\vec{S}^{k_{j}, k}$ is a normal $(p+1)$-series, we have by SIH that $k \leq M\left(p+1, k_{j}\right)$. On the other hand we have $M(p+1, n)=M(p+$ $1, M(p, n))$ by Definition $18 r_{k_{j}} \geq r_{k_{0}}=r_{n}$ and $e_{p}(M(p, n))<_{\varepsilon_{0}} e_{p}(n)$, Lemma 17. Hence $k \leq M\left(p+1, k_{j}\right)=M(p+1, n)$.

2. $k_{j}<M(p, n)$ :

(a) $j=l$ : Then $k_{l}<M(p, n)$, and hence $k=k_{l}+1 \leq M(p, n) \leq$ $M(p+1, n)$.

(b) $j<l$ : Since $\vec{S}^{k_{j}, k_{j+1}}$ is a normal $p$-series, we have $k_{j+1} \leq M\left(p, k_{j}\right)$ by MIH. On the other hand we have $M\left(p, k_{j}\right) \leq M(p, n)$ by (4). Thus $k_{j+1} \leq M(p, n)$, and this is not the case. 
19]3. Let $H=H(C r):=M(p, 0 ; C r)$ for $p:=\mathrm{RANK}-1$. If $S^{0}$ is solving, then $0=H$. Suppose that $S^{0}$ is nonsolving. By Lemma $1911 \vec{S}^{0, H}$ is a $p$-series. From Lemma 16 and $r_{0}=0 \leq r_{H}$ we see that $\vec{S}^{0, H+1}$ is a $(p+1)$-series. But this means that $\vec{S}^{0, H+1}$ is a $p$-series by Lemma 1513c. Therefore we see from Lemma 1912 that $\vec{S}^{0, H+1}$ is not normal, i.e., $S^{H}$ is solving.

Lemma 20 The function $(p, n, C r) \mapsto M(p, n ; C r)$ is $\varepsilon_{0}$-recursive.

Proof. It suffices to see that $M(p, n ; C r)$ is defined by nested recursion on the ordinal $\varepsilon_{0}$. Then it is $\varepsilon_{0}$-recursive by a result in W. W. Tait $\left[9\right.$ and $\omega^{\varepsilon_{0}}=\varepsilon_{0}$.

Suppressed the parameter $C r$, let us define a function $M^{\prime}(p, n, y)$ as follows: $M^{\prime}(0, n, y):=n+1$.

Case $0 S^{n}$ is solving: $M^{\prime}(p+1, n, y):=n$.

Case $1 S^{n}$ is nonsolving.

$$
\begin{aligned}
& \text { 1. } o\left(\vec{S}^{M^{\prime}(p, n, y), M^{\prime}\left(p, M^{\prime}(p, n, y), y\right)}\right) \nless_{\varepsilon_{0}} y \text { : } \\
& M^{\prime}(p+1, n, y):=M^{\prime}(p, n, y) . \\
& \text { 2. } r_{M^{\prime}(p, n, y)}<r_{n} \& o\left(\vec{S}^{M^{\prime}(p, n, y), M^{\prime}\left(p, M^{\prime}(p, n, y), y\right)}\right)<_{\varepsilon_{0}} y \text { : } \\
& M^{\prime}(p+1, n, y):=M^{\prime}(p, n, y) . \\
& \text { 3. } r_{M^{\prime}(p, n, y)} \geq r_{n} \& o\left(\vec{S}^{M^{\prime}(p, n, y), M^{\prime}\left(p, M^{\prime}(p, n, y), y\right)}\right)<_{\varepsilon_{0}} y \text { : } \\
& M^{\prime}(p+1, n, y):=M^{\prime}\left(p+1, M^{\prime}(p, n, y), o\left(\vec{S}^{M^{\prime}(p, n, y), M^{\prime}\left(p, M^{\prime}(p, n, y), y\right)}\right)\right) .
\end{aligned}
$$

Then $M^{\prime}(p, n, y)$ is seen to be defined by nested recursion on the lexicographic ordering $\prec$ on pairs $(p, y):(p, y) \prec(q, z)$ iff $p<q$ or $p=q \& y<\varepsilon_{0} z$. 18.

Then $M(p, n):=M^{\prime}\left(p, n, \omega_{\mathrm{RANK}+2}\right)$ enjoys the defining clauses in Definition

\section{Exact bounds: infinite ranks}

In this section let us compute the length of the H-process for theories of jump hierarchies, which is slightly modified from 2 .

The normal function $\theta_{\alpha}: \beta \mapsto \theta \alpha \beta$ is the $\alpha^{\text {th }}$ iterate of the function $\theta 1 \beta=$ $\omega^{\beta}$. Fix an ordinal $\Lambda<\Gamma_{0}$, the least strongly critical number, and let $<_{\Gamma_{0}}$ denote a standard primitive recursive well ordering of type $\Gamma_{0}$ with the least element 0 . In what follows the subscript in $<_{\Gamma_{0}}$ is omitted.

Let $\mathcal{A}(x, \alpha, z, X)$ be a fixed quantifier free formula in the language of first order arithmetic. Let $(H)_{\Lambda}$ denote the theory of the absolute jump hierarchy $\left\{H_{\alpha}\right\}_{\alpha \leq \Lambda}$ generated by the formula $\mathcal{A}$ and up to $\alpha \leq \Lambda$ :

$$
\alpha \leq_{\Gamma_{0}} \Lambda \rightarrow\left\{y \in H_{\alpha} \leftrightarrow \exists x \mathcal{A}\left(x, \alpha, y, H_{<\alpha}\right)\right\}
$$


where $H_{<\alpha}=\sum_{\beta<\alpha} H_{\beta}$, i.e., $H_{<\alpha}$ denotes the binary abstract $\{(\beta, z):(\beta, z) \in$ $\left.H_{<\alpha}\right\}$.

The critical formulas in its $\epsilon$-counterpart are the $\epsilon$-axiom (1),

$$
\alpha \leq \Lambda \rightarrow\left\{t \in H_{\alpha} \leftrightarrow \mathcal{A}\left(\epsilon x \mathcal{A}\left(x, \alpha, t, H_{<\alpha}\right), \alpha, t, H_{<\alpha}\right)\right\}
$$

and

$$
\alpha \leq \Lambda \rightarrow\left\{(\beta, t) \in H_{<\alpha} \leftrightarrow \beta<\alpha \wedge t \in H_{\beta}\right\}
$$

Now the rank of an expression is defined such that $r k(e)<3 \Lambda+\omega$. For a given finite sequence $C r$ of critical formulas, let RANK $=\operatorname{RANK}(C r)=3 \Lambda+n>$ $r k(C r):=\max \left\{r k\left(C r_{I}\right): I=0, \ldots, N\right\}$ for an $n<\omega$.

An $\epsilon$-substitution is a finite function assigning numerical values $|\epsilon x . F|_{S} \in \omega$ to canonical terms $\epsilon x . F$, and boolean values $|e|_{S}=\top$ for expressions $e$ in one of the shapes $n \in H_{\alpha}$ or $(\beta, n) \in H_{<\alpha}$ such that $\beta<\alpha \leq \Lambda$.

Define the Ackermann ordering $<_{A}$ and $\|x\|_{A}$ as in (2), where for boolean values, $\perp<_{A} \top$ and $\|\perp\|_{A}=0,\|\top\|_{A}=1$.

Define the index $\operatorname{ind}(S)<\operatorname{IND}=\operatorname{IND}(C r)=(\omega+1)^{N(C r)}<\omega^{\omega}$ of $S$ relative to a fixed sequence $\mathrm{Cr}$ of critical formulas as in Definition 4 .

\subsection{Bounds on $p$}

Since RANK $\geq \omega$, Lemma $1513 \mathrm{C}$ is useless here. We need to give a bound on $p$ such that $M(p, n ; C r)<M(p+1, n ; C r)$.

Let $\ell_{p}=M(p+1,0 ; C r) .\left\{\ell_{p}\right\}$ is an increasing sequence $\ell_{p} \leq \ell_{p+1}$, and once $\ell_{p}=\ell_{p+1}$, then $\ell_{p}=\ell_{q}$ for any $q \geq p$.

Now let $p(C r)$ denote the least number $p$ such that $\ell_{p}=\ell_{p+1}$ if such a $p$ exists. We show that the number $p(C r)$ is defined. Then $S^{H}$ is a solution of $C r$ for $H=M(p(C r), 0 ; C r)$.

For each $p$, let $\vec{S}_{p+1}=\left\{S^{n}\right\}_{n<\ell_{p}}$ be the $(p+1)$-section according to Lemma 1911, and $\vec{S}_{p+1}=\vec{S}_{p}^{1} * \cdots * \vec{S}_{p}^{l_{p}}$ its decomposition into $p$-sections $\vec{S}_{p}^{j}$.

Let $o(\vec{S})=o(\vec{S} ; 0)<\theta(\mathrm{RANK})(\mathrm{IND})<\theta \Lambda \varepsilon_{0}$ denote the ordinal associated to sections $\vec{S}$ as in [2].

Then let $\alpha_{p}^{j}=o\left(\vec{S}_{p}^{j}\right)$ for $0<j<l_{p}$, and $\alpha_{p}^{0}:=\theta(\mathrm{RANK})(\mathrm{IND})$.

By Lemma 17 we have

$$
\alpha_{p}^{j}>\alpha_{p}^{j+1}
$$

Moreover let $\gamma_{p}^{j}=r_{k_{p}^{j+1}}\left(0 \leq j<l_{p}\right)$, where $S^{k_{p}^{j}}$ is the first term in the substring $\vec{S}_{p}^{j+1}$ for $j<l_{p}$, and $k_{p}^{l_{p}}=\ell_{p}$.

Finally let for $\Delta:=$ RANK $>\gamma_{p}^{j}$

$$
\theta \Lambda \varepsilon_{0}>\beta_{p}:=\sum_{j<l_{p}} \Delta^{\alpha_{p}^{j}} \gamma_{p}^{j}=\Delta^{\alpha_{p}^{0}} \gamma_{p}^{0}+\cdots+\Delta^{\alpha_{p}^{l_{p}-1}} \gamma_{p}^{l_{p}-1}
$$

From (5) we see that $\beta_{p}$ is in Cantor normal form. 
Lemma $21 \beta_{p}>\beta_{p+1}$ if $\ell_{p}<\ell_{p+1}$.

Proof. Assume $\ell_{p}<\ell_{p+1}$. This means that $S^{\ell_{p}}$ is nonsolving.

By Lemma 192 we have $\gamma_{p}^{l_{p}-2}>\gamma_{p}^{l_{p}-1}$.

Let

$$
J=\min \left\{j \leq l_{p}-2: \gamma_{p}^{j}>\gamma_{p}^{l_{p}-1}\right\} .
$$

Then

$$
k_{p}^{J}=\max \left\{i<\ell_{p}: r_{i} \leq \gamma_{p}^{l_{p}-1}\right\}
$$

and by Lemma 13 we have $\vec{S}_{p+1}^{j}=\vec{S}_{p}^{j}$ for $0<j \leq J$. Hence $\alpha_{p+1}^{j}=\alpha_{p}^{j}$ for $0 \leq j \leq J$, and $\gamma_{p+1}^{j}=\gamma_{p}^{j}$ for $0 \leq j<J$.

Consider the next substitution $S^{\ell_{p}}$ to the last one in $\vec{S}_{p}^{l_{p}}$ or equivalently the last one in $\vec{S}_{p+1}$. Then $S^{\ell_{p}}$ is the next substitution to the last one in $\vec{S}_{p+1}^{J+1}$ by Lemma 13

Hence $\gamma_{p}^{J}>\gamma_{p}^{l_{p}-1}=\gamma_{p+1}^{J}$. Therefore $\beta_{p}>\beta_{p+1}$ as desired.

Now $p(C r)=F(0, C r)$ for the function

$$
F(p, C r)= \begin{cases}F(p+1, C r) & \text { if } \ell_{p+1}>\ell_{p} \\ \min \left\{q \leq p: \ell_{q+1}=\ell_{q}\right\} & \text { otherwise }\end{cases}
$$

$F(p, C r)$ is defined by a $\theta \Lambda \varepsilon_{0}$-external recursion by Lemma 21,

\section{Exact bounds: impredicative cases}

Let $\mathrm{T}$ denote one of the theories $\Phi$-FIX for non-monotonic inductive definitions for the formula classes $\Phi=\Pi_{1}^{0},\left[\Pi_{1}^{0}, \Pi_{1}^{0}\right], \Pi_{2}^{0}$ (cf. [3], [4], [5] and [7]).

$O(\mathrm{~T})$ denotes the system of ordinal diagrams for $\mathrm{T}$. T is a two sorted theory: one sort for natural numbers, and the other sort for ordinals. The well ordering $<$ on ordinals is understood to be the ordering in the notation system $O(\mathrm{~T})$, when the values of expressions are calculated. Its largest value is denoted $\pi \in$ $O(\mathrm{~T})$, which is intended to be a closure ordinal of non-monotonic inductive definitions by the operators in $\Phi . \Omega \leq \pi$ is the first non-recursive ordinal $\omega_{1}^{C K}$. $d_{\Omega} \varepsilon_{\pi+1}$ denotes the proof-theoretic ordinal of $\mathrm{T}$, and the length of the H-process should be calculated by $d_{\Omega} \varepsilon_{\pi+1}$-recursion.

The rank of an expression is defined such that $r k(e)<\pi+\omega$. For a given finite sequence $\mathrm{Cr}$ of critical formulas, let RANK $=\mathrm{RANK}(\mathrm{Cr})=\pi+n(\mathrm{Cr})$ for an $n(C r)<\omega$ so that $\max \left\{r k\left(C r_{I}\right): I=0, \ldots, N\right\}<$ RANK. Then $\pi \neq r k(S)<$ RANK for any $S$ appearing in the H-process for $C r$.

Define the index $\operatorname{ind}(S)<\operatorname{IND}=\operatorname{IND}(C r)=(\pi+1)^{N(C r)}<\pi^{\omega}$ of $S$ relative to a fixed sequence $C r$ of critical formulas as in Definition 4.

In Definition $18 M(p, n)$ is defined by $\pi^{\omega}$-recursion, i.e., $o(\vec{S})<\pi^{\omega}$, and $p(C r)$ is defined by $\varepsilon_{\pi+1}$-recursion, i.e., $o(\vec{S})<\varepsilon_{\pi+1}$, which are far from $d_{\Omega} \varepsilon_{\pi+1}<$ $\Omega<\pi^{\omega}<\varepsilon_{\pi+1}$.

For the moment, suppose that $M(p, n)$ has been defined for each $p<\omega$. Let $\ell_{p}=M(p+1,0 ; C r)$. 
If $\exists p \leq n(C r)\left[\ell_{p}=\ell_{p+1}\right]$, then there is nothing to prove, i.e., $p(C r) \leq n(C r)$. In what follows assume $\forall p \leq n(C r)\left[\ell_{p}<\ell_{p+1}\right]$, and let $p>n(C r)$. Suppose $\ell_{p}<\ell_{p+1}$. Then $S^{\ell_{p}}$ is nonsolving.

Consider the number

$$
m_{p}:=\max \left\{n<\ell_{p}: r_{n}<\pi\right\}
$$

Note that $m_{p}$ is in the set $\operatorname{nd}\left(\vec{S}^{0, \ell_{p}}\right)$.

Proposition $22 p>n(C r) \& \ell_{p}<\ell_{p+1} \Rightarrow \pi>r_{m_{p}}>r_{\ell_{p}}$.

Proof of Proposition 22, Suppose $p>n(C r), \ell_{p}<\ell_{p+1}$ and $r_{m_{p}} \leq r_{\ell_{p}}$. By Lemma 1944 we have $M(p, n) \leq M(p+1, n) \leq M(p+1,0)=\ell_{p}$ for any $n<\ell_{p}$. If $M\left(p, m_{p}\right)=\ell_{p}$, then we would have $M(p+1, n)>\ell_{p}$ by $r_{m_{p}} \leq r_{\ell_{p}}$. Hence $n_{p}:=M\left(p, m_{p}\right)<\ell_{p}$. On the other hand we have $r_{n_{p}}>\pi>r_{m_{p}}$, and hence $M\left(p, m_{p}\right)<M\left(p+1, m_{p}\right) \leq \ell_{p}$. From this we see that $\vec{S}^{m_{p}, M\left(p+1, m_{p}\right)}$ is a proper $(p+1)$-series. Therefore a component in the decomposition of $\vec{S}^{m_{p}, M\left(p+1, m_{p}\right)}$ is a proper $p$-series. On the other side any component except the first one is an improper $p$-series by $r_{n}>\pi\left(m_{p}<n<\ell_{p}\right)$, Lemma 15]3b and $p \geq n(C r)$. Hence the first component $\vec{S}^{m_{p}, m^{\prime}}\left(m^{\prime} \leq M\left(p, m_{p}\right)\right)$ is a proper $p$-series. But

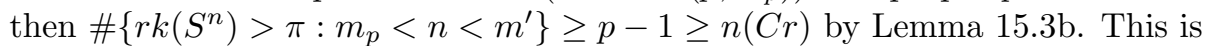
a contradiction. We have shown Proposition 22 .

For each $p>n(C r)$, let $\vec{S}_{p+1}=\left\{S^{n}\right\}_{n<\ell_{p}}$ be the $(p+1)$-section. Decompose $\vec{S}_{p+1}$ into $\vec{S}_{p+1}=\vec{S}_{p}^{1} * \cdots * \vec{S}_{p}^{l_{p}-1} * \vec{S}_{p}^{l_{p}}$ where the last substring $\vec{S}_{p}^{l_{p}}$ is defined to be $\vec{S}^{m_{p}, \ell_{p}}$ for the number $m_{p}$ in (6), and each $\vec{S}_{p}^{j}=\vec{S}_{p}^{k^{j-1}, k_{p}^{j}}\left(1 \leq j<l_{p}\right)$ for $\left\{(0=) k_{p}^{0}, k_{p}^{1}, \ldots, k_{p}^{l_{p}-1}\left(=m_{p}\right)\right\}_{<} \subseteq \mathrm{nd}\left(\vec{S}_{p+1}\right)$. Put $k_{p}^{l_{p}}=\ell_{p}$.

Let $o(\vec{S})=o(\vec{S} ; 0)<d_{\Omega} \omega_{n(C r)}(\pi)$ denote the ordinal associated to $p$-series $\vec{S}$ as in [3], 4, [5] and [7]. Note the following fact:

$\alpha<\omega_{n(C r)}(\pi)$ for any subdiagram $d_{\sigma}^{q} \alpha$ occurring in ranks, indices and

$o(\vec{S})$, which appear in the H-process for $\mathrm{Cr}$

Then let $\alpha_{p}^{j}=o\left(\vec{S}_{p}^{j}\right)$ for $0<j \leq l_{p}-1$, and $\alpha_{p}^{0}:=d_{\Omega} \omega_{n(C r)}(\pi)$.

We have, cf. Theorem 11.7 in [3]

$$
\Omega>\alpha_{p}^{j}>\alpha_{p}^{j+1}
$$

Let $\gamma_{p}^{j}:=r_{k_{p}^{j+1}}\left(0 \leq j<l_{p}\right)$ and $\Delta:=\pi>\gamma_{p}^{j}$. Finally let

$$
\beta_{p}:=\omega_{n(C r)}(\pi)+\sum_{j<l_{p}} \Delta^{\alpha_{p}^{j}} \gamma_{p}^{j} .
$$

Lemma $23 d_{\Omega} \beta_{p} \in O(\mathrm{~T})$, and $d_{\Omega} \beta_{p}>d_{\Omega} \beta_{p+1}$ if $\ell_{p}<\ell_{p+1}$ and $p>n(C r)$. 
Proof. To show $d_{\Omega} \beta_{p} \in O(\mathrm{~T})$, we have to verify a condition $\mathcal{B}_{>\Omega}\left(\beta_{p}\right)<\beta_{p}$ for a set $\mathcal{B}_{>\Omega}\left(\beta_{p}\right)$ of subdiagrams of $\beta_{p}$. This is seen from (7) and $\mathcal{B}_{>\Omega}\left(\beta_{p}\right)=$ $\mathcal{B}_{>\Omega}\left(\left\{\gamma_{p}^{j}: j<l_{p}\right\}\right)$.

Assume $\ell_{p}<\ell_{p+1}$ and $p>n(C r)$. Let

$$
J=\min \left\{j \leq l_{p}-2: \gamma_{p}^{j}>\gamma_{p}^{l_{p}-1}\right\} .
$$

Note that by Proposition 22 we have $r_{m_{p}}=\gamma_{p}^{l_{p}-2}>\gamma_{p}^{l_{p}-1}=r_{\ell_{p}}$.

Then as in the proof of Lemma21 we see $\alpha_{p+1}^{j}=\alpha_{p}^{j}$ for $0 \leq j \leq J, \gamma_{p+1}^{j}=\gamma_{p}^{j}$ for $0 \leq j<J$. Moreover we have $\gamma_{p}^{J}>\gamma_{p}^{l_{p}-1}=\gamma_{p+1}^{J}$. Therefore $\beta_{p}>\beta_{p+1}$.

It remains to show $K_{\Omega} \beta_{p+1}<d_{\Omega} \beta_{p}$. By (8) it suffices to see $K_{\Omega}\left\{\gamma_{p+1}^{j}: j<\right.$ $\left.l_{p+1}\right\}<d_{\Omega} \beta_{p}$.

If $\mathrm{T}=\Phi$-FIX for $\Phi=\left[\Pi_{1}^{0}, \Pi_{1}^{0}\right], \Pi_{2}^{0}$, then there is nothing to prove, i.e., $K_{\Omega}\left\{\gamma_{p+1}^{j}: j<l_{p+1}\right\}=\emptyset$.

Consider the case $\Phi=\Pi_{1}^{0}$ and $\pi=\Omega$. Then we have $K_{\Omega}\left\{\gamma_{p+1}^{j}: j<l_{p+1}\right\}<$ $\alpha_{p}^{0}=d_{\Omega} \omega_{n(C r)}(\Omega)<d_{\Omega} \beta_{p}$ by (17).

It remains to define $M(p, n)$ by $d_{\Omega} \varepsilon_{\pi+1}$-recursion. This is seen from the following lemma.

Lemma 24 Let $\vec{S}^{i}=\vec{S}^{m_{i}, k_{i}}(i=0,1)$ be two consecutive p-series, $k_{0}=m_{1}$. Assume $r_{m_{1}} \geq r_{m_{0}}$. Then $o\left(\vec{S}^{1}\right)<o\left(\vec{S}^{0}\right)$.

Proof. This is seen as in Lemma 17, i.e., Theorems 10.8 and 11.7 in 3 .

Thus we have shown that both $M(p, n ; C r)$ and $p(C r)$ are defined by $d_{\Omega} \varepsilon_{\pi+1^{-}}$ recursion. This yields a solution $S^{H}$ of $C r$ for $H=M(p(C r), 0 ; C r)$.

\section{References}

[1] Ackermann, W.: Zur Widerspruchsfreiheit der Zahlentheorie. Math. Ann. $117,162-194(1940)$

[2] Arai, T.: Epsilon substitution method for theories of jump hierachies, Arch. Math. Logic 41, 123-153 (2002)

[3] Arai, T.: Epsilon substitution method for $I D_{1}\left(\Pi_{1}^{0} \vee \Sigma_{1}^{0}\right)$, Ann. Pure Appl. Logic 121, 163-208 (2003)

[4] Arai, T.: Epsilon substitution method for $\left[\Pi_{1}^{0}, \Pi_{1}^{0}\right]$-FIX, Arch. Math. Logic 44, 1009-1043(2005)

[5] Arai, T.: Ideas in the epsilon substitution method for $\Pi_{1}^{0}$-FIX, Ann. Pure Appl. Logic 136, 3-21(2005)

[6] Arai, T.: An expository survey on epsilon substitution method, in Proceedings of the Asian Mathematical Congress, Singapore (2005) 
[7] Arai, T.: Epsilon substitution method for $\Pi_{2}^{0}$-FIX, Jour. Symb. Logic 71, $1155-1188(2006)$

[8] Tait, W. W.: Functionals defined by transfinite recursion, Jour. Symb. Logic 30, 155-174(1965)

[9] Tait, W. W.: Nested recursion, Math. Ann. 143, 236-250(1961) 